\title{
Using High-Fidelity Simulation to Teach Ethics Related Non-Technical Skills: Description of an Innovative Model
}

This article was published in the following Dove Press journal:

Advances in Medical Education and Practice

\section{Issam Tanoubi $\mathbb{D}$ \\ L Mihai Georgescu \\ Arnaud Robitaille \\ Pierre Drolet \\ Roger Perron}

Department of Anesthesiology, Centre d'apprentissage des Attitudes et Habiletés Cliniques (CAAHC), Université de Montréal, Faculty of Medicine, Montreal, Quebec, Canada
Correspondence: Issam Tanoubi $M D, M A(E d)$, DESAR, Department of Anesthesiology, Centre d'apprentissage des Attitudes et Habiletés Cliniques (CAAHC), Université de Montréal, Faculty of Medicine, Montreal, Quebec, Canada

Email i.tanoubi@umontreal.ca

\begin{abstract}
This article describes a high-fidelity (Hi-Fi) simulation-based innovative educational strategy intended to introduce anesthesiology residents to key ethical considerations and how they apply to their practice. Three Hi-Fi simulation scenarios involving situations with various ethical issues are described with their debriefing objectives and the trainees' subjective feedback. Three high-fidelity simulation scenarios are described: (a) teaching critical incident disclosure, (b) disclosing and discussing patient awareness during general anesthesia, and (c) would physicians override a do-not-resuscitate (DNR) order if the cause of a cardiac arrest is iatrogenic? We used Hi-Fi simulation in an innovative way to teach these principles of ethics. Simulation, through carefully crafted debriefing, can contribute to the acquisition of essential non-technical ethical skills. How best to integrate simulation in an existent ethics curriculum and how it compares with more traditional teaching methods are questions that need to be addressed.
\end{abstract}

Keywords: high-fidelity simulation, ethics principles education, ethic scenario, debriefing

\section{Introduction}

This article describes a high-fidelity (Hi-Fi) simulation-based innovative educational strategy intended to introduce anesthesiology residents to key ethical considerations and how they apply to their practice. Three Hi-Fi simulation scenarios involving situations with various ethical issues are described, with their debriefing objectives and the trainees' subjective feedback.

\section{Teaching Critical Incident Disclosure}

It is estimated that $50 \%$ of healthcare professionals will be exposed at least once in their career to a critical incident involving patient care. While disclosing such events is both a deontological and in most jurisdictions a legal responsibility, several obstacles such as distressing emotions, fear of litigation, or lack of proper critical communication skills may prevent individuals from adequately fulfilling this obligation. ${ }^{1}$ Thus, for medical educators, it becomes an ethical and pedagogical obligation to properly train their students and equip them with the necessary tools required for the accomplishment of such duties.

This report describes how anesthesiology trainees, who rarely interact with family members of patients and mostly perform short-term patient follow-ups, can use HiFi simulation to elicit a reflective process and learn a strategy for disclosing an intraoperative critical incident. 
A Hi-Fi simulation-based training session was designed by a team of instructors (AR, MG, IT and RP: Initials of 4 of the instructors among the authors of the manuscript) to re-enact the unexpected intra-operative death of a healthy young parturient due to a massive amniotic fluid embolism. A group of four residents participated in each session. Accompanied by one faculty member playing the role of an operating room nurse and two of the students acting as surgeon and respiratory therapist, the two main participants played the role of anesthesiologists, one as the primary caregiver in charge of the case (hot seat) and the other who could be called for help, if needed (cold seat).

In the scenario, following the uneventful cesarean delivery of a healthy baby, the mother experienced acute desaturation, combined with significant and refractory hypotension. Over a period of $15 \mathrm{mins}$, despite adequate diagnosis and management, her condition progressed to pulseless electrical activity, followed by pulseless ventricular tachycardia, ventricular fibrillation, and finally asystole. The case evolved toward refractory and terminal asystole, prompting the surgical and anesthetic teams to jointly decide that resuscitation maneuvers should be stopped. A faculty member acting as a consultant anesthetist was scheduled to intervene on an as-needed basis to support the team in its decision to stop resuscitation efforts.

During the structured debriefing, beyond the technical review of amniotic fluid embolism management and resuscitation efforts, which were secondary goals of the session, several aspects pertaining to the main objectives were covered. Firstly, the emotions caused by the events were explored, through an open discussion that included perception, potential misconceptions and implications with regard to medical errors. Emphasis was placed on the fact that iatrogenic morbidity and mortality, when present, are often multifactorial and firm conclusions regarding their causes should be drawn only after a thorough investigation of the event, and not in the immediate aftermath. Secondly, barriers to appropriate disclosure of the event, mandatory in the immediate aftermath, were explored. The discussion was oriented towards fear of litigation, with a reassurance to the trainees that adequate disclosure would most likely contribute to a reduction in the severity of legal implications, while the opposite may be true about inadequate disclosure. ${ }^{1}$ Finally, a multiple-step strategy regarding the disclosure of adverse events ${ }^{2,3}$ was proposed and discussed with the trainees, which included:
1. Immediate internal debriefing of the surgical and anesthetic teams aimed at the normalization of emotions and completion of medical records with the reporting of as many facts as possible.

2. Analysis of the suspected causes of the adverse event, avoiding framing it in terms of medical error or attribution of individual responsibility for the outcome.

3. Preparation for the disclosure of the event to family members by a multidisciplinary team representing all the individuals involved. It was suggested to seek professional supportive care, both for family members and staff.

4. Emphasis on the communication of facts, avoiding suppositions or conclusions about uncertain causes, events, or attribution of responsibility.

The training was positively received by the students, and they gave high scores in the immediate, post-training satisfaction assessment, regarding their satisfaction and the impact of the simulation on their subsequent practice. A weakness of our curriculum is that due to time constraints, practicing disclosure procedures with mock family members could not be undertaken.

\section{Disclosing and Discussing Patient Awareness During General Anesthesia}

Another scenario was designed to introduce junior anesthesia residents ( 3 or 4 residents in each session) to systemic factors leading to medical errors and undesirable outcomes and, once more, to the divulgation of such errors once they have occurred.

The two-part scenario first involved creating a situation in which multiple systemic factors resulted in patient awareness during general anesthesia and then having the anesthesia team practice disclosing and discussing the event with an actor playing the patient.

The first part of the scenario took place in a Hi-Fi simulation suite mimicking an operating room. During a laparoscopic appendicectomy performed under general anesthesia on an obese man (Hi-Fi mannequin), multiple elements combined to distract the anesthesia personnel who then became unaware of an inadvertent reduction in the depth of anesthesia, causing intraoperative patient awareness. The scenario began with a confederate playing the role of a respiratory therapist calling the resident in the hot seat and seeking help since he could not find the attending anesthesiologist. The participant then 
entered the operating room with the crisis ongoing and without the benefits of a proper briefing. The operating room lights had been dimmed for laparoscopy, making visual assessment of the situation more difficult, and the surgeon, another confederate, experienced technical difficulties, prompting him to make inappropriate comments about the patient's obesity, and to rudely complain about inadequate patient paralysis. The surgeon incessantly asked questions to the resident in the hot seat, thereby interrupting his train of thought. The respiratory therapist was fixated on a problem with patient ventilation - peak airway pressure being high and oxygen saturation being low, pointing to the patient's endotracheal tube and telling the resident that the tube had probably migrated endobronchially due to the steep Trendelenburg position requested by the surgeon. The respiratory therapist kept insisting that the resident should auscultate the chest, which was difficult, given that access was limited by the presence of surgical drapes and that the surgeon was constantly talking and complaining about the operating conditions. In this scenario, the confederate playing the respiratory therapist was asked to do all he could to transfer his fixation to the anesthesia resident. Whenever the resident looked towards the monitor and anesthesia machine, the respiratory therapist and the surgeon diverted his attention elsewhere.

It was then expected, because of the aforesaid factors, that the anesthesia resident would be distracted enough to be unaware of the decreasing level of anesthesia that was leading to the patient becoming aware. During most sessions, the participant playing the resident was so engrossed with the multiple distractors that he allowed the patient to become severely hypertensive while waking up. The first portion of the scenario was to end with the resident deepening the anesthesia after he realized that it was too shallow.

During the debriefing, participants typically began by blaming themselves for not noticing that anesthesia was not deep enough. However, using the model of human error developed by James Reason to analyze the situation, ${ }^{4,5}$ the debriefer was to shift the focus from the individual to the system and have the participants identify the various systemic factors that were parts of the scenario as contributing agents in the crisis.

At the end of the first debriefing session, the debriefer announced that a few days had passed following the surgery and that the patients had requested to see his anesthesiologist. The patient, played by an actor, met the participant (the attending anesthesiologist initially in charge of anesthesia was said to be unavailable) and told him that he remembered parts of the surgery. The actor was instructed not to elaborate initially on his experience and its psychological impact, to check whether the participant would try to elicit this information on his own. If the participant did not, the actor was to tell the resident he remembered having been called "fat" (we used an overweight actor). Similarly, the actor did not initially ask whether an error had occurred, to see what the participant would disclose. Later, if the resident failed to freely start disclosing relevant facts, the actor was to directly question the participant, "Why did this happen?", "Am I normal?" and "Could this happen again?". This second part of the scenario was also followed by a debriefing session during which, after allowing participants to vent their emotions, the debriefer generally highlighted how awareness typically presents itself, ${ }^{6}$ using the patient's experience as a starting point. Error disclosure, in particular its medicolegal and ethical aspects, was also discussed. ${ }^{7}$ This scenario was positively perceived with a major estimated impact on the subsequent practice of residents.

\section{Would Physicians Override a Do-Not- Resuscitate (DNR) Order if the Cause of a Cardiac Arrest Is latrogenic?}

The last scenario dealt with a situation in which administering a medication in the wrong way led to a cardiovascular arrest in a patient who had earlier clearly expressed her wish not to be resuscitated. An IV patient-controlled analgesia device containing morphine and an epidural analgesic local anesthetic infusion were both installed in the same patient for pain management, following a laparotomy for removal of a digestive neoplasia. Unfortunately, the similarity of the two infusion pumps had led to the devices being mistakenly switched. The patient then received, through the epidural route, the morphine intended to be administered intravenously and vice versa for the local anesthetics. The error led to a cardiovascular arrest and the three residents who were quickly summoned to the scene had to decide whether or not they should initiate cardiopulmonary resuscitation (CPR). It was made clear to them that the patient's explicit wish was not to be resuscitated in the event of a cardiac arrest. Whether the DNR order also applied to a situation resulting from a medication error, was never discussed.

In all the sessions, each team participating in the scenario tacitly came to a consensus that CPR should be continued. Following each session, an ethics expert acted as a co-debriefer in order to help answering the following 
question, should physicians override a DNR order when a cardiac arrest is iatrogenic?

Though this situation may be unusual, it has been reported on numerous occasions and many experts have voiced their opinions regarding its ethical aspect have been written. ${ }^{8-10}$ The decision made by the participants in this particular scenario was undoubtedly influenced by contextual elements. Physicians may assume that DNR orders do not or should not apply to iatrogenic cardiac arrests. They may conclude that patients, having neither discussed nor envisaged a situation where the arrest was iatrogenic, would have made an exception for such situation. A physician may also believe that since the condition is iatrogenic, it creates an implicit obligation to provide treatment, maybe even more so if the error was committed by the physician himself. ${ }^{9}$ Also, the fact that factors associated with some iatrogenic cardiac arrest may increase the likelihood of successful CPR with no neurologic damage may lead the physician to assume that had the patient had known he could expect a positive outcome, he would have agreed to be reanimated. In situations when CPR has a foreseeable higher likelihood of success, patients may express preferences for treatment and it could be stated in a DNR order. Indeed, a generic DNR order issued without discussion of foreseeable situations in which "higher-success" CPR can be expected may not accurately convey the patient's wish. ${ }^{10}$ Since an iatrogenic arrest is not usually included in the range of events anticipated by patients when discussing a DNR order, it could be argued that they did not actually consent to it being withheld. Some authors then concluded that since there is no true informed refusal of treatment, the patient should be resuscitated. ${ }^{11}$

The debriefing after this scenario highlighted the major arguments to override the DNR order: the principle of nonmaleficence, the efficacy of resuscitation, proximate cause, and physician error. These arguments were balanced with the fact that overriding the DNR order did not respect the patient's autonomy, decision, and goals. The physician should not have a moral choice but to follow the DNR order. Emphasis was put on the need for better communication between physicians and patients regarding advance care planning and better documentation of these discussions. ${ }^{12}$

\section{Discussion}

High-fidelity simulation creates a physical, emotional and contextual clinical environment close to reality. ${ }^{13}$ The ultimate goal is to create an immersive experience which, if reproduced in real life, would unfold in similar sequences and outcomes and will offer the same learning whether the scenario was simulated or actual. Simulation would thus allow to adequately cohere to the principle of Kolb's experience learning. ${ }^{14}$ The high fidelity depends mainly on the learning objective and not only on the physical support used for the simulation. ${ }^{15}$ The scenarios chosen were aimed at a technical clinical objective (such as resuscitation of a cardio-circulatory arrest or anesthetic induction) requiring the use of advanced technological support (mannequin and monitors) and nontechnical communication objectives (announcement of bad news, decision not to resuscitate) requiring confederate actors to play specific roles (family, nurse). Our scenarios offered specifically oriented high fidelity.

The Holy Grail of medical simulation is the proof of clinical transferability of teaching, with an impact on patient outcome. While publications have begun to accumulate bringing this kind of evidence, ${ }^{16,17}$ proof is still relatively scarce. Yet, as David Gaba mentioned a few years back ${ }^{18,19}$ we cannot afford to wait for hard evidence before implementing a method of training which intuitively has the potential for significant clinical benefits. This is particularly true in cases of rare and severe complications, such as the ones we address in the simulation sessions described, where traditional training methods can only offer theoretical preparation. We have no hard evidence that such training has a positive clinical impact, but resident satisfaction surveys and oral comments indicate they feel the approach we employed has enriched their training and raised their confidence in addressing such distressing, but extremely rare, clinical events. We have received this quality of feedback over more than ten cohorts of residents ( 24 residents in each cohort), spanning more than 10 years of high-fidelity simulation. We hence feel confident that our training approach complements traditional training methods positively for these subjects.

\section{Conclusion}

We used Hi-Fi simulation in an innovative way to teach some ethics principles. Simulation, through carefully crafted debriefing, can contribute to the acquisition of essential non-technical ethical skills. How best to integrate simulation in an existent ethics curriculum and how it compares with more traditional teaching methods, are questions that need to be addressed.

\section{Disclosure}

None of the authors have any competing interests in this work. 


\section{References}

1. Larouche C. La divulgation d'un événement indésirable et les mots pour le dire. Le médecin du Québec. 2013;(48):85-87.

2. Aitkenhead AR. Anaesthetic disasters: handling the aftermath. Anaesth. 1997;52(5):477-482. doi:10.1111/ana.1997.52.issue-5

3. Garnier E. Divulgation des événements indésirables. comment y faire face? comment s'y prendre? Le médecin du Québec. 2013(48):21-27.

4. Reason J. Human error: models and management. BMJ. 2000;320 (7237):768-770. doi:10.1136/bmj.320.7237.768

5. Reason J. Safety in the operating theatre - part 2: human error and organisational failure. Qual Saf Health Care. 2005;14(1):56-60.

6. Pandit JJ, Andrade J, Bogod DG, et al. 5th national audit project (NAP5) on accidental awareness during general anaesthesia: summary of main findings and risk factors. Br J Anaesth. 2014;113 (4):549-559. doi:10.1093/bja/aeu313

7. Windwick B, Aubin D, Beard P, et al. Canadian Disclosure Guidelines. Canadian Patient Safety Institute. In: InstituteCPS,editors. 2011, Available from:https://www.patientsafetyinstitute.ca/en/ toolsResources/disclosure/Documents/CPSI\%20 Canadian\% 20Disclosure\%20Guidelines.pdf. Accessed March 24, 2020.

8. Casarett D, Ross LF. Overriding a patient's refusal of treatment after an iatrogenic complication. $N$ Engl J Med. 1997;336(26):1908-1910. doi:10.1056/NEJM199706263362611

9. Casarett DJ, Stocking CB, Siegler M. Would physicians override a do-not-resuscitate order when a cardiac arrest is iatrogenic? $J$ Gen Intern Med. 1999;14(1):35-38. doi:10.1046/j.1525-1497.1999.00278.x

10. Choudhry NK, Choudhry S, Singer PA. CPR for patients labeled DNR: the role of the limited aggressive therapy order. Ann Intern Med. 2003;138(1):65-68. doi:10.7326/0003-4819-138-1-20030107000014
11. Hall SA. Overriding a patient's refusal of treatment after an iatrogenic complication. $N$ Engl J Med. 1997;337(20):1477.

12. Ross LF. Do not resuscitate orders and iatrogenic arrest during dialysis: should "no" mean "no"? Semin Dial. 2003;16(5):395-398. doi:10.1046/j.1525-139X.2003.16081.x

13. Nanji KC, Baca K, Raemer DB. The effect of an olfactory and visual cue on realism and engagement in a health care simulation experience. Simul Healthc. 2013;8(3):143-147. doi:10.1097/ SIH.0b013e31827d27f9

14. Schultz K, McEwen L, Griffiths J. Applying Kolb's learning cycle to competency-based residency education. Acad Med. 2016;91(2):284. doi:10.1097/01.ACM.0000479943.21563.6f

15. Rudolph JW, Simon R, Raemer DB. Which reality matters? questions on the path to high engagement in healthcare simulation. Simul Healthc. 2007;2(3):161-163. doi:10.1097/SIH.0b013e31813d1035

16. Issenberg SB, McGaghie WC, Petrusa ER, Lee Gordon D, Scalese RJ. Features and uses of high-fidelity medical simulations that lead to effective learning: a BEME systematic review. Med Teach. 2005;27(1):10-28. doi:10.1080/01421590500046924

17. Okuda Y, Bryson EO, DeMaria S Jr., et al. The utility of simulation in medical education: what is the evidence? Mt Sinai J Med. 2009;76 (4):330-343. doi:10.1002/msj.v76:4

18. Dutta S, Gaba D, Krummel TM. To simulate or not to simulate: what is the question? Ann Surg. 2006;243(3):301-303. doi:10.1097/01. sla.0000200853.69108.6d

19. Paige JT, Terry Fairbanks RJ, Gaba DM. Priorities related to improving healthcare safety through simulation. Simul Healthc. 2018;13(3S Suppl 1):S41-S50. doi:10.1097/SIH.0000000000000295
Advances in Medical Education and Practice

\section{Publish your work in this journal}

Advances in Medical Education and Practice is an international, peerreviewed, open access journal that aims to present and publish research on Medical Education covering medical, dental, nursing and allied health care professional education. The journal covers undergraduate education, postgraduate training and continuing medical education

\section{Dovepress}

including emerging trends and innovative models linking education, research, and health care services. The manuscript management system is completely online and includes a very quick and fair peer-review system. Visit http://www.dovepress.com/testimonials.php to read real quotes from published authors. 